\title{
Las necesidades de información en la comunidad de vitiviniculto- res de la región de Baja California: una aproximación.
}

\author{
Fermín López Franco *
}

Artículo recibido:

6 de mayo de 2010.

Artículo aceptado:

9 de agosto de 2010.

\section{RESUMEN}

Los vitivinicultores manifiestan necesidades de información y comportamiento informativo, pero no conocen los aspectos de este proceso. Por ejemplo, no se tiene la certeza del lugar y las fuentes de información a las que acuden a fin de llevar a cabo sus diferentes actividades como son la selección de la uva de acuerdo con la zona, la siembra, el cuidado, la cosecha, producción y comercialización de la vid, además del embotellamiento de sus productos.

En este artículo se presentan algunos aspectos relacionados con la definición de las necesidades de información, así como una breve descripción de éstas en el área agrícola y biológica al no encontrar información

* Universidad Nacional Autónoma de México. fermin|@servidor.unam.mx

INVESTIGACIÓN BIBLIOTECOLÓGICA, Vol. 24, Núm. 51, mayo/agosto, 2010, México, ISSN: 0187-358X. pp. 125-145 
que se relacione directamente con los vitivinicultores. La metodología fue una investigación documental y de campo, mientras que la técnica que se empleó fue una encuesta que utilizó como instrumento la cédula de entrevista. Además se presentan algunos resultados obtenidos relacionados con necesidades de información de esta comunidad.

Palabras clave: Necesidades de Información; Vitivinicultura; México; Baja California.

\section{ABSTRACT}

An approach to information needs of a community of winemakers in baja california

Fermin López Franco

Winemakers have information needs about their discipline, including matters regarding grape selection, planting and care, harvest, production and commercialization. Moreover, they exhibit behaviors associated with meeting these needs; however the processes and information sources involved are not known. This paper provides definitions of these information needs and a brief contextualization of this information within the larger fields of agriculture and biology when information specifically associated with winemaking is unavailable. The methodology involves documentary research, field study, surveys and interviews. A description of some results regarding the information needs of winemakers is also presented.

Key words: Information needs; Winemakers; México; Baja California.

\section{INTRODUCCIÓN}

as necesidades de información dice Calva son las "manifestaciones de re- 
o de más alto nivel, en las cuales intervienen los factores ambientales externos". 1

Según lo expresado en el párrafo anterior las necesidades de información surgen cuando el ser humano ha cubierto sus necesidades básicas y requiere de información para llevar a cabo sus actividades cotidianas.

También Calva afirma que "el término necesidades de información es una construcción genérica usada para responder por qué las personas buscan, encuentran y utilizan la información". 2

Se considera entonces que las necesidades de información surgen del individuo al reconocer una carencia, en este caso de información, la cual será utilizada para llevar a cabo una determinada actividad, que puede ser de investigación, de docencia, laboral o escolar.

Otro autor, Ashill, define a las necesidades de información como aquellas características de la información que involucran un comportamiento y se refieren a la calidad de la información, y que tienen una utilidad en la toma de decisiones. ${ }^{3}$

De lo expresado anteriormente surgió la inquietud por identificar las necesidades de información de la comunidad de vitivinicultores, en este caso aquellos que se ubican en Ensenada, en particular los del valle de San Antonio de las Minas y de Guadalupe.

La comunidad de vitivinicultores tienen necesidades de información y su manifestación es desconocida para los bibliotecólogos, en general se desconocen los temas que requieren conocer para desarrollar las diversas fases de sus actividades, las cuales van desde la selección de la vid, la siembra, el cuidado, la cosecha, la vinificación, el embotellado y la promoción de sus productos.

La importancia de investigar esta comunidad y su entorno radica en que es esto un sector significativo en México, que se ubica en diversas regiones donde existen casas productoras de vino que representan, en materia de empleos, aproximadamente

1 Juan José Calva González, "Las necesidades de información de los usuarios en la planeación bibliotecaria”, en Biblioteca Universitaria: Boletín Informativo de la Dirección General de Bibliotecas, Vol. 6, Núm. 1, 1991, p p. 25-30.

2 Juan José Calva González, Las necesidades de información: su naturaleza, su manifestación y detección, México, El autor, 1988, 247 p. (Tesis de Maestría), UnAM, Facultad de Filosofía y Letras.

3 Nicholas J. Ashill, "Defining the information needs of senior marketing executives: an exploratory study", en Qualitative Market Research: An International Journal, Vol. 4, Núm. 1, 2001, p. 53 . 
9 mil 500 fuentes de trabajo, repartidas en 4 mil 500 empleos directos (incluidas las fábricas, oficinas y laboratorios de producción) y casi 5 mil de jornales (campo vitícola, manuales y otros servicios). ${ }^{4}$

México es el productor más antiguo de vino en América, pero su industria de vinos de calidad es relativamente reciente; en este aspecto, el país enfrenta una fuerte competencia ante sus vecinos del norte (Estados Unidos) y del sur (Argentina y Chile). En las otras bebidas alcohólicas como el tequila, el pulque y el mezcal, todavía domina México la producción.

Los viñedos más grandes e importantes de la República Mexicana se ubican en el área sur de la línea fronteriza con Estados Unidos desde Mexicali hasta Ensenada.

\section{NeCesidAdes De InFormación}

Antes de comenzar a definir las necesidades de información es importante comentar los objetivos e hipótesis que se desarrollaron para hacer el presente trabajo. Los objetivos eran los siguientes:

- Establecer las variables que motivan la aparición de las necesidades de información de los vitivinicultores de Vitis vinifera para su producción y comercialización.

- Identificar las necesidades de información de los productores vitivinicultores en la región de Baja California en las cuales se cultiva la Vitis vinifera.

- Identificar las unidades de Información a las que acuden los productores de la vid para satisfacer sus necesidades de información y comportamiento.

Mientras que la hipótesis relacionada con las necesidades de información fue la siguiente:

- La información sobre normas, regulaciones, situación geográfica (clima, suelo) es un factor externo que determina el trabajo de los productores vitivinícolas en México con respecto a la siembra, cosecha, producción y comercialización, con el fin de mantenerse en el mercado 
competitivo tanto a nivel nacional como internacional, con los demás productores nacionales y extranjeros.

Además, era necesario precisar el significado de las necesidades de información o requerimientos de información que manifestaban las personas, comunidades de alumnos, profesores, investigadores y sectores productivos, entre otros; por lo que a continuación se presentan algunas definiciones de este término para comprender mejor el fenómeno.

Se consideran necesidades de información aquellas que

...surgen de una motivación para buscar, recuperar y usar la información, la cual tiene un carácter eminentemente utilitario y de producción. Aún para los investigadores que aparentemente utilizan información sin fines prácticos e inmediatos, esta utilización está fundamentada en la necesidad de producir conocimiento o simplemente para apoyar la toma de decisiones. ${ }^{5}$

La necesidad de información es definida por Sanz Casado como "la sensación de la carencia de algo". 6

Por otro lado se dice que las "necesidades de información" son necesidades en su más alto nivel; es decir, tras haber satisfecho las necesidades de alimento, vestido, etcétera, el ser humano empieza a tener conciencia o raciocinio, y esto lo hace sentir la necesidad de conocer lo que sucede a su alrededor.

Calva considera a las necesidades humanas como las

manifestaciones de reacciones que tiene el individuo, ya sean por causas básicas o fisiológicas, o de más alto nivel, en las cuales intervienen los factores ambientales externos. ${ }^{7}$

Concluye diciendo que las necesidades de información surgen cuando se han cubierto las necesidades básicas.

Cabe recordar que una necesidad de información es un término creado artificialmente para expresar que las personas requieren de información para desarrollar sus diferentes actividades.

5 Terminología relacionada con los estudios de usuarios, en Seminario Latinoamericano sobre Formación de Usuarios de la Información y Estudios de Usuarios, México, CUIB, 1997, p. 3.

6 Elías Sanz Casado, Manual de estudios de usuarios, Madrid, Fundación Germán Sánchez Ruipérez, 1994, p. 24.

7 Juan José Calva González, "Las necesidades de información de los usuarios en la planeación bibliotecaria", en Biblioteca Universitaria: Boletín Informativo de la Dirección General de Bibliotecas, Vol. 6, Núm. 1, 1991, pp. 25-30. 
Las necesidades de información surgen de una motivación para buscar, recuperar y usar la información la cual tiene un carácter eminentemente utilitario y de producción. Aun para los investigadores que aparentemente utilizan información sin fines prácticos e inmediatos, ésta utilización está fundamentada en la necesidad de producir conocimiento, o simplemente para apoyar la toma de decisiones. ${ }^{8}$

Con lo anterior se puede reafirmar que el concepto de "necesidades de información" es un término abstracto construido expresamente para identificar lo que un individuo a manera personal, profesional, etcétera, requiere para satisfacer su carencia de información y así obtener el o los documentos que demanda para compensar dicha necesidad.

Un aspecto que no debe pasar inadvertido son las características propias de los usuarios, es decir algunos de los elementos que los conforman y que, como dice Calva: "se puede identificar y agrupar a los usuarios con base en otras características o combinación de ellas": ${ }^{9}$

- Profesión.

- Edad.

- Raza.

- Posición social.

- Sexo.

- Ocupación.

- Lugar de residencia.

- Nivel educativo.

- Lugar de trabajo.

Con lo anterior se sintetizan los factores internos y externos que hacen posible el surgimiento de las necesidades de información, y se destacan la profesión del individuo y las relaciones que tenga con sus colegas, así como la edad que le proporciona experiencia para identificar y manejar las fuentes de referencia afines a su disciplina, por lo que respecta a la raza, posición social, sexo, ocupación, y los demás factores que motivan las manifestaciones necesitadas de información.

Por otro lado Devadason menciona que las necesidades de información documental se ven afectadas por una serie de factores como:

- El rango de las fuentes disponibles.

8 “Terminología relacionada con los estudios de usuarios", en Seminario Latinoamericano sobre Formación de Usuarios de la Información y Estudios de Usuarios, México, CUIB, 1997, p 3. 
- La retroalimentación.

- La motivación.

- La orientación profesional.

- Las características individuales del usuario.

- Los sistemas que rodean al usuario: social, político, económico, legal y regulador.

- Las consecuencias del uso de la información. ${ }^{10}$

Pero una necesidad de información ¿cómo o por qué surge? Santos afirma que

aparecen cuando el ser humano ya ha cubierto sus necesidades básicas, tales como alimento, de manera aceptable y se encuentra influenciado por factores externos como su medio ambiente, el medio político, económico, educativo, etcétera; los cuales harán que tenga una reacción al haber cubierto sus necesidades básicas. ${ }^{11}$

El hecho de identificar las necesidades de información en profundidad permitirá desarrollar una colección de materiales documentales cuyo contenido deberá responder con efectividad a las necesidades de información de la comunidad a la que sirve; es por ello que desde un inició al planear los servicios bibliotecarios deben identificarse los factores internos y externos que conforman esta comunidad para que la biblioteca adecue sus servicios a dichas características.

Harris menciona que utilizará la expresión "estudios de usuarios”, en lugar del término "necesidades de los usuarios". Los estudios de usuarios dice Harris

son el conjunto de estudios relacionados con las necesidades de información de los individuos o de grupos y su comportamiento en la búsqueda y uso de la información. ${ }^{12}$

La definición proporcionada por Harris engloba dentro del término "estudios de usuarios” a las necesidades de información y al comportamiento,

10 J. W. Devadason, “A methodology for identification of information needs of users", en IFLA Journal, Vol. 23, Núm. 1, 1997, p. 42.

11 Antonia Santos Rosas y Calva González, Juan José, "Identificación de las Necesidades de información de los usuarios: un estudio", en Revista : Documentación de Ciencias de la Información, No. 20, 1997, pp. 207-224.

12 Colin Harris, "Necesidades de los usuarios y mercado de la información: su importancia en el currículo de las escuelas de bibliotecología”. - En: Revista Interamericana de Bibliotecología. Vol. 9, No. 1, 1986. - p. 15 
pero no menciona ninguna característica respecto a ambos tópicos; es más el autor incluye dentro de los "estudios de usuarios" una parte del mercadeo que se denomina "Investigación de Mercados", que no incluye ni promoción de servicios, ni guía o instrucción sobre su uso.

Castillo Sánchez menciona que las necesidades de información del usuario pueden ser:

a) Necesidad de un único documento u obra para solucionar determinado interés.

b) Necesidad de obtener uno o más documentos sobre un tema determinado.

c) Necesidad de una investigación exhaustiva. ${ }^{13}$

Con las definiciones proporcionadas se puede reafirmar que el concepto de "necesidades de información" es un término abstracto construido expresamente para identificar lo que un individuo, de manera personal, profesional, etcétera, requiere para satisfacer su carencia de información y así obtener el o los documentos que demanda para satisfacer esa necesidad.

\section{NECESIDADES DE INFORMACIÓN EN LA VITIVINICULTURA}

No se han encontrado estudios sobre las necesidades y el comportamiento informativo de la comunidad de vitivinicultores en un país determinado. Se han ubicado trabajos que tratan sobre las necesidades y el comportamiento informativo en el área de la agricultura y sobre las necesidades de información de los campesinos.

Las ciencias biológicas y agrícolas son áreas muy cercanas. Las primeras examinan aspectos fundamentales de los sistemas vivos y su relación con el medio ambiente, mientras que las ciencias agrícolas se enfocan a aspectos nacionales y globales de la producción y el consumo de alimentos. ${ }^{14}$

Entre otros autores que han estudiado el tema, se encuentra Pali Kuruppu, cuyas investigaciones tienen por objetivo examinar las necesidades de información de científicos del área de la biología y la agricultura en la Universidad estatal de Iowa, relacionadas con la enseñanza, la investigación y las actividades de extensión.

13 Octavio Castillo Sánchez, "Técnicas de recolección de información más utilizadas en los estudios de usuarios”, en Boletín del Departamento de Bibliotecología, Núm. 16 (1987), p. 48.

14 Pali U. Kuruppu, "Understanding the information needs of academic scholars in agricultural and biological sciences", en The Journal of Academic Librarianship. Vol. 32, Núm. 6, 2006, p. 609. 
Los cuestionamientos de los que parte Kuruppu para llevar a cabo su trabajo son los siguientes:

- ¿Cuál es el tipo de información que utilizan los científicos de las áreas biológica y agrícola?

- ¿Qué fuentes utilizan ambas comunidades?

- ¿Qué herramientas y procedimientos utilizan para localizar su información?

- ¿Qué fuentes y servicios adicionales les gustaría tener disponibles para encontrar su información ${ }^{15}$

En los cuestionamientos que Kuruppu menciona se incluyen las necesidades y el comportamiento en la búsqueda de información, y se mencionan los motivos por los cuales los investigadores requieren las fuentes a las que acuden para localizar su información.

Por otra parte Lisa Nason sostiene que los aspectos específicos que requieren los granjeros son los siguientes: ${ }^{16}$

- Información sobre métodos de agricultura.

- Legislación y políticas sobre operaciones agrícolas.

- Políticas de mercado.

Al analizar el trabajo de los dos autores se puede observar que parten de preguntas similares con el fin de obtener información sobre lo que requieren los científicos del área biológica y agrícola, la cual se traduce en información relacionada con métodos en agricultura, legislación y políticas de comercialización, aspectos que se relacionarán más adelante al mencionar los resultados obtenidos por el presente trabajo.

\section{El CUltivo DE LA VID}

El cultivo de la vid para la producción del vino es una de las actividades más antiguas de nuestra civilización, y probablemente contemporánea al comienzo de ésta. Existe evidencia de que los primeros cultivadores de viñas y productores de vino vivían en la región de Egipto y Asia Menor, durante el neolítico. Al mismo tiempo que los primeros asentamientos humanos permanentes

15 Ibid. p. 611.

16 Lisa Nason, "The farmers in the library: information needs of farmers and how the rural public library can fulfill them”, 19-45 pp., en Rural Library, Vol. 27, Núm. 2, 2007, p. 20. 
empezaban a dominar el arte del cultivo y la cría de ganado, así como la producción de cerámica.

La uva es el fruto de las plantas del género Vitis, perteneciente a la familia de las vitáceas. Las especies originarias de las zonas templadas del hemisferio norte incluyen variedades utilizadas como frutos de mesa, pasas, vinos y zumos de uva o mostos. ${ }^{17}$

La especie comúnmente usada en la obtención del vino es la Vitis vinifera procedente de Eurasia donde apareció hace miles de años, como lo demuestra la presencia de fósiles de esta planta en distintos yacimientos prehistóricos; tiene raíz honda, gruesa, leñosa, tronco gris pardusco de madera porosa de hasta $1,7 \mathrm{~m}$ de circunferencia, tenaz y flexible y las hojas pecioladas.

Es importante destacar que en el argot de los vitivinicultores se entiende por vendimia a la recolección o cosecha de las uvas de vino; y que en el caso de las uvas de mesa se usa simplemente el término cosecha. El periodo de vendimia varía entre febrero y abril (en el hemisferio Sur), y julio y octubre (en el hemisferio norte).

El proceso de la vendimia depende del grado de maduración de la uva que se desee; es decir, del momento en que la relación porcentual entre los azúcares y los ácidos en el grano de uva han alcanzado el valor óptimo para el tipo de vino que se desea producir. Si bien este parámetro es genéricamente válido para la uva de mesa, en el caso de la uva destinada a la producción de vino es necesario considerar otros parámetros para decidir cuándo es el tiempo de vendimia. ${ }^{18}$

Al cultivo de la uva se le denomina viticultura, una práctica antigua que se remonta hasta la época del neolítico. Jeroglíficos egipcios del tercer milenio anterior a la era cristiana, citas bíblicas y referencias a ella en las obras homéricas confirman la realidad del consumo del vino como hecho habitual, ${ }^{19}$ mientras que la vinicultura se refiere al proceso de elaboración de vinos. ${ }^{20}$

Por lo que respecta a la enología se dice que es el "conjunto de conocimientos relativos a la elaboración de los vinos". En las estaciones enológicas se estudian y clasifican las diversas variedades de uva, se practican análisis y se hacen los estudios necesarios para conocer los mostos y los vinos que resultarán, también se efectúan mezclas para formar tipos de aceptación en el mercado y se estudian las enfermedades de la vid y de los vinos.

17 Enciclopedia Hispánica, Barcelona, 2ª . Ed., Editorial Barsa-Planeta, 2003, T. 14, p. 214.

18 Restaurantes de Mexico.com.mx La vendimia: una tradición de la industria vinícola, http:// www.restaurantesdemexico.com.mx/379/Vinos_y_Bebidas_La_Vendimia__Una_Tradicion_En_La_Industria_Vinicola.html\# 6-ago-2008.

19 Enciclopedia Hispánica, Barcelona, 2a. Ed., Editorial Barsa-Planeta, 2003, T. 14, p. 214.

20 Enciclopedia universal ilustrada: europea americana, Madrid, Espasa-Calpe, 1996, T. 14, p. 4661. 


\section{Vitivinicultura en MÉXico}

Cabe repetir que México es el productor americano más antiguo de vino, pero su industria de vinos de calidad es relativamente reciente. En cuanto a otras bebidas como el tequila y el mezcal entre otros, todavía domina la producción mexicana, pero en cuanto al vino el país enfrenta una fuerte competencia ante sus vecinos del norte (Estados Unidos) y del sur (Argentina y Chile).

Corresponde a Hernán Cortés el mérito de haber sido el primer promotor del cultivo de la Vitis vinifera en México, el primer sitio del continente americano donde comenzó a ser cultivada regularmente la vid. El 20 de marzo de 1524 -otros dicen que el 24 de marzo del mismo año-Cortés firmó las Ordenanzas de buen gobierno para los vecinos y moradores de la Nueva España. Luis Hidalgo, enólogo español, afirma que estas Ordenanzas se hallan en el Archivo del Duque de Terranova y Monteleone, en el Hospital de Jesús, de la ciudad de México. ${ }^{21}$

En México son 14 los estados que se dedican a la producción de uva, entre los que destacan: Sonora, Zacatecas, Baja California, Aguascalientes y Coahuila; los cuales, durante el periodo de 1997 a 2007, contribuyeron con el $97.7 \%$ de la superficie sembrada a nivel nacional. ${ }^{22}$

La península de Baja California con 1,257 km. de largo y un promedio de $90 \mathrm{~km}$. de ancho, que la convierte en una de las penínsulas más grandes del mundo, tiene casi el $12 \%$ de las aguas territoriales de México, y posee también $1,381 \mathrm{~km}$. de playa a lo largo del Océano Pacífico, y $640 \mathrm{~km}$. a lo largo del Golfo de California (Mar de Cortés).

El estado de Baja California está situado en la región noroeste en la semidesierta península del mismo nombre en la que sólo brota la vegetación gracias a la humedad de las nieblas matutinas y las brisas marinas. El clima es mediterráneo con inviernos húmedos y veranos secos y templados. ${ }^{23}$

Baja California es uno de los estados más importantes de México en lo que a producción agrícola y pesca se refiere. Hoy cuenta además con una creciente industria de procesamiento de alimentos.

Entre los productos alimenticios bajacalifornianos destacan: ${ }^{24}$

21 Miguel Guzmán Peredo, El vino en México, http://www.mexicodesconocido.com.mx/ notas/744-El-vino-en-Méxiso, 2-feb-2009.

22 Sistemas Producto: Perspectivas del mercado de la uva, http://www.campomexicano.gob. $\mathrm{mx} /$ portal_sispro/index.php?portal=uva, 26-sep-2008.

23 Luis Tomás Melgar Gil, La enciclopedia del vino, Madrid, Ramón Llaca, 2008. p. 70.

24 Baja California: la frontera Mexicana para negocios de éxito, http://www.investinbaja.gob. $\mathrm{mx} /$ industrias/alimentos.htm, 27-sep-2008. 
- Hortalizas: brócoli, espárrago, cebollín, tomate, fresas, dátiles, etcétera.

- Pescados y mariscos: atún, macarela, sardina, almeja, mejillón, langosta, camarón y abulón.

- Bebidas: jugos de frutas, refrescos, cerveza y vinos.

Especial mención merece la industria vitivinícola, ya que la producción de Baja California equivale a más del $90 \%$ del vino producido en el país. Muchos de estos productos, por su alta calidad, son comercializados en los exigentes y sofisticados mercados de Norteamérica, Japón y la Unión Europea.

Las variedades blancas que se cultivan en esta zona son:

- Riesling

- Chardonnay

- Chenin Blanc

- Moscatel

- Gewurztraminer

- Sauvignon Blanc

- Colombard

- Palomino

Mientras que dentro de las uvas tintas se cultivan las siguientes:

- Cabernet Sauvignon

- Anglianico

- Cabernet Franc

- Grenache

- Barbera

- Nebbiolo

- Merlot

- Pinoit Noir

- Zinfandel

- Tempranillo

- Semillón

- Syrah

Los jesuitas plantaron las primeras vides en las misiones de Baja California desde 1697 y fueron reemplazados por los franciscanos cuando en 1767 se los expulsó de América. Finalmente los Dominicos continuaron con esa actividad, abriendo nuevas misiones y cultivos en los valles de Guadalupe y Santo Tomás. 
Se ha elaborado vino en Baja California desde el siglo XVIII, al traer los misioneros jesuitas a la península sarmientos de lo que después se conocería como uva misión y que hasta la fecha sigue cultivándose. Sin embargo, fue hasta el año de 1834 cuando los misioneros dominicos establecieron la Misión de Nuestra Señora de Guadalupe del Norte, la cual le dio su nombre al valle. Esta fue la última misión establecida en las Californias y la que menos tiempo funcionó, pues fue abandonada en 1840 después de un ataque indígena. ${ }^{25}$

\section{Ruta DEL VINO}

Ubicada en la parte norte del estado de Baja California, la Ruta del Vino goza del clima Mediterráneo ideal para el cultivo de la vid. Hay pocos microclimas en México que combinen esas características únicas de altitud, tierra, temporadas, tiempo y temperatura. Sin olvidar el amor al arte de hacer vino. ${ }^{26}$

Los valles de San Antonio de las Minas y de Guadalupe son el corazón de la Ruta del Vino, a continuación se presenta una breve reseña de cada uno de estos valles:

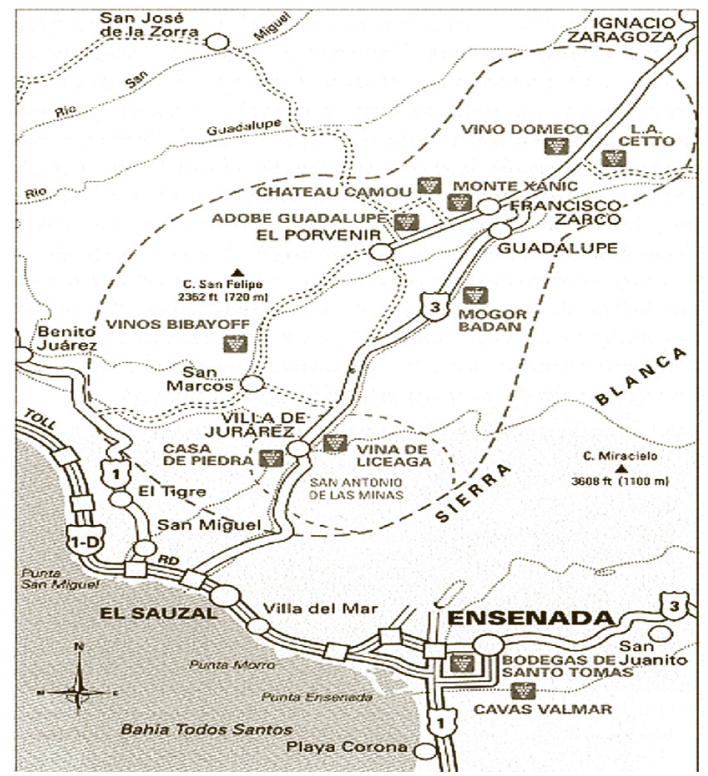

25 Monte Xanic, http://www.montexanic.com.mx/inicio.html 2-ago-2008.

26 Baja California: la frontera mexicana para negocios de éxito: Ruta del vino, http://www.investinbaja.gob.mx/estilo/recreacion.htm\#06 27-sep-2008. 


\section{San Antonio de las Minas}

Es un pequeño pueblo de 494 habitantes ubicado a 240 metros de altitud y considerado el corazón y uno de los principales atractivos de la llamada ruta del vino, misma que se extiende también hacia el norte al Valle de las Palmas y hacia el sur a los de Santo Tomás y San Vicente Ferrer, lugares en donde se cultivan uvas Chenin Blanc, Colombard, Sauvignon Blanc y Chardonnay para los vinos blancos, al igual que Cabernet Sauvignon, Merlot, Cabernet Franc, Grenache, Carignan, Barbera, Nebbiolo y Zinfandel para los tintos. En el pueblo se pueden visitar los Vestigios de las Minas de San Antonio, las cuales dieron origen a este poblado en el siglo XIX; asimismo, en el museo, ubicado en la calle principal, puede uno deslumbrarse con los restos de una compañía minera abandonada del siglo XIX. ${ }^{27}$

\section{Valle de Guadalupe}

El Valle de Guadalupe es una región vitivinícola localizada en el estado de Baja California, México. Se le conoce equivocadamente también como Valle de Calafia, nombre que se ha intentado usar para promover los productos de esta región a nivel nacional e internacional; sin embargo, el nombre correcto es Valle de Guadalupe, establecido gracias a la misión que los franciscanos establecieran en una de sus mesetas, la Misión de Nuestra Señora de Guadalupe del Norte", misma que los indígenas nativos destruyeran en el año de 1840 aproximadamente. ${ }^{28}$

El Valle de Guadalupe es un lugar excepcional que se encuentra en la vertiente occidental de la península enclavado en el suave declive de las montañas hacia la costa del Pacífico; a esta tierra de clima cálido y seco llega la brisa y la neblina oceánica, lo que le confiere un ambiente mediterráneo. El invierno es frío y lluvioso debido a los vientos monzónicos del noroeste, y el verano cálido debido a los vientos del sureste, lo que brinda un clima propicio para que la vid crezca y madure. ${ }^{29}$

Hasta aquí se ha tratado de proporcionar una visión general de las necesidades de información en la vitivinicultura, y aunque no se ha encontrado información relacionada específicamente con esta área ha sido posible ubicar trabajos que tratan sobre requerimientos informativos de agricultura y los campesinos.

27 Caminata mensual a San Antonio de las Minas en Ensenada, http://bajacalifornia.travel/caminata-mensual-a-san-antonio-de-las-minas-en-ensenada/ 1-sep-2008.

28 Valle de Guadalupe en Wikipedia, http://es.wikipedia.org/wiki/Valle_de_Guadalupe_(Baja_California) 30-junio-2008

29 Vinos L. A, Cetto: vinos con nombre y apellido, http://www.lacetto.com/28-ago-2008. 
Asimismo se esbozó una breve reseña de la uva y de los aspectos relacionados con ella, particularmente una sucinta semblanza del desarrollo del cultivo de la vid en la región de Baja California, especialmente en los valles de San Antonio de las Minas y de Guadalupe.

Al tener comunicación con la Asociación de Vitivinicultores de Baja California se pudieron obtener los datos de los productores de vino de dicha zona para contactarlos y realizar la investigación de sus necesidades de información.

\section{Metodología}

Para la realización de este trabajo se llevó a cabo:

- Una investigación documental y el análisis crítico de las fuentes encontradas.

- Una Investigación de campo en la región norte del estado de Baja California.

La técnica que se empleó fue la siguiente:

- Entrevista dirigida a los productores de Vitis vinifera.

El instrumento que se utilizó para la presente investigación fue la:

- Cédula de entrevista:

La aplicación de la entrevista se llevó a cabo con 14 vitivinicultores.

El motivo por el cual se seleccionó la entrevista es que se quiso tener la oportunidad de hablar con los responsables de las vinícolas y obtener datos relevantes relacionados con la industria, ya que es una técnica flexible y permite la posibilidad de aclarar las preguntas. Al tener contacto personal con ellos y darles la posibilidad de sentirse en confianza obtuvimos más información de la que esperábamos, ya que algunos de ellos les gustó el hecho de ser objeto de estudio y esto nos permitió indagar mejor acerca de la información que requieren ellos para llevar a cabo sus actividades.

La cédula de entrevista se estructuró en dos partes:

- Datos personales como nombre del entrevistado, edad, profesión, puesto en la empresa y años en la misma, relación con el viñedo y cómo 
nace su interés por la industria del vino.

- Información que necesitan sobre las actividades de procedimiento, cultivo, recolección, aspectos empresariales y en general del mercado del vino; y también sobre las fuentes y recursos que utilizan o a los que acuden para obtenerla.

\section{ANÁLISIS DE RESULTADOS}

Las respuestas que proporcionaron las personas entrevistadas en la región de Baja California, en particular los valles de San Antonio de las Minas y de Guadalupe son las siguientes:

1) Por lo que respecta a la primera parte de la cédula se obtuvieron los siguientes datos:

- Ingeniero agrónomo, ayudante del viticultor, enólogo principal, ayudante del enólogo, agricultor, maestro normalista, bibliotecario, biólogo.

- La edad de los entrevistados fluctúa de los 26 hasta los 56 años.

- Años en la organización; manifestaron de 4 hasta 55 años, pero hay quienes contestaron "toda mi vida".

- La relación que tienen con el viñedo tiene que ver con la cosecha, el encargado del viñedo, el dueño, el socio, el enólogo.

- Acerca de cómo nació su interés por la vitivinicultura comentaron que fue por herencia de sus padres y abuelos, una cuestión personal.

2) En lo concerniente a la segunda parte de la cédula se pudo recabar la siguiente información:

- Procedimiento:

- Las personas entrevistadas realizan diversas actividades en el viñedo; es decir, el cuidado de la planta, la cosecha, la vinificación, la venta de sus productos. En pocos casos las personas entrevistadas tienen sólo el cargo de ser los responsables del viñedo.

- La información que obtienen para realizar su trabajo les llega a través de la experiencia, acuden a proveedores de insumos, agrónomos, enólogos, etcétera.

- Acuden a fuentes como distribuidores, catálogos de venta, páginas de Internet, personas del medio, colegas, asesores, 
conferencias, libros, revistas, la experiencia de otros.

- Mencionan que de todas las actividades que realizan ninguna es más fácil o difícil que la otra y cuando no pueden llevarla a cabo acuden a otra persona que les pueda apoyar en la realización de ella.

- Cultivo:

- Los problemas que surgen en el cultivo de la vid, los resuelven acudiendo al agrónomo, al enólogo, pero en su mayor parte resuelven los problemas a través de la experiencia.

- El número de hectáreas de cultivo de la vid lo determinan de acuerdo con el proyecto que tienen en mente.

- La forma de cultivar la vid o expandir el viñedo depende de su experiencia y la realiza por medio de estacas, reducir el tamaño del surco, etcétera.

- La variedad de uva que se va a cultivar está de acuerdo con el proyecto que van a realizar, o a la uva que han sembrado para otra vinícola.

- La información para darle alta calidad al vino es solicitada al enólogo, y depende de su experiencia.

- La información que se requiere para el cultivo es en su mayor parte facilitada por el enólogo, o personas que ya han realizado actividades de vendimia.

- Algunas de las personas entrevistadas manifiestan comprar uvas para completar su producción.

- Recolección:

- Los viticultores saben cuánta gente requieren recurriendo a su experiencia, además de que las personas contratadas ya han realizado la actividad con anterioridad.

- La maquinaria es en su mayoría mecánica y sólo en pocos casos manual, y para realizar las reparaciones mayores en el caso de la maquinaria mecánica se recurre a personal especializado; en pocos casos el personal de la vinícola realiza las reparaciones.

- Empresa:

- El nombre de la empresa es definido casi siempre por el apellido del o de los dueños, un recuerdo familiar o algún nombre en una lengua indígena.

- Por sus lecturas en revistas, libros e Internet saben de otros productores de vino en el país y en el mundo.

- Generalmente coinciden en afirmar que para establecer el 
precio de su vino es pertinente cubrir sus costos de producción; asimismo sólo unos cuantos consideran la producción del vino como un arte.

- Cuentan con asesores para el pago de impuestos y para comprender las leyes que rigen a la industria vinícola.

- En su mayoría cuentan con intermediarios para vender sus productos, aunque ellos mismos hacen muchas veces dicha actividad.

- Consideran a sus colegas como no competidores; a decir de ellos, "si le va bien a uno, nos va bien a todos".

- Dependiendo del tipo de empresa, grande o pequeña, en el primero de los casos se va ampliando su mercado, en el de los pequeños vitivinicultores éstos piensan únicamente en conservar al menos el mercado que tienen.

- Acuden a ferias y exposiciones para dar a conocer sus productos.

Lisa Nason, señala que

los agricultores en el desarrollo de las naciones tienen necesidades similares a las de sus colegas norteamericanos, como son el tipo de suelo de los campos de cultivo, el clima, y necesitan acceder a tecnología agrícola, obtención de créditos y mercado de precios. ${ }^{30}$

Algunos aspectos informativos que menciona Lason y que requieren los vitivinicultores de esta región de México son los siguientes:

- Información sobre métodos de agricultura.

- Legislación y políticas agrícolas operacionales.

- Políticas de mercado.

- Controles de plagas.

- Procedimientos de cultivo.

- Novedades en técnicas de siembra.

- Vinificación.

- La industria del vino en el mundo.

- Historia de los valles de San Antonio de las Minas y de Guadalupe. blic library can fulfill them”, 9-45 pp., en Rural Library, Vol. 27, Núm. 2, 2007, p. 20. 
En este último párrafo se puede apreciar que los vitivinicultores manifiestan necesidades de información similares a las de los agricultores, como alude Nason.

\section{Conclusiones}

Los vitivinicultores de la región de Baja California manifiestan sus necesidades de información en forma similar a los que mencionan Kuruppu y la propia Lason, requieren información sobre métodos de agricultura, legislación y políticas agrícolas.

Por lo aquí expresado se puede observar que los vitivinicultores requieren información sobre nuevos métodos de cultivo, cuestiones legislativas y aspectos relacionados con la mercadotecnia, todo lo cual es necesario para mantener la producción y calidad del producto.

Esta información puede servir de base para atender a esta comunidad, o bien, se pueden realizar estudios sobre los vitivinicultores de otras regiones del país, además de mostrar que los estudios de usuarios se pueden aplicar a las diversas comunidades y no sólo a sectores académicos y estudiantiles.

Es imprescindible y necesario que la disciplina bibliotecológica replique este tipo de estudios entre otros sectores sociales con el fin de apoyarlos a satisfacer sus necesidades de información; haciendo esto se apoyará de manera fehaciente al desarrollo económico, cultural y social de las diversas comunidades atendidas, como fue el caso en el sector vitivinicultor al incorporar, con estos trabajos de investigación, nuevos conocimientos al proceso de la siembra, cosecha, elaboración, manufactura y comercialización de sus productos vinícolas.

Por lo que respecta a las fuentes de información relacionadas con las necesidades de información en la comunidad de vitivinicultores, ésta fue difícil de localizar porque no se ubicaron artículos que tuvieran dicha información, y porque se hallaron pocos artículos relacionados con las necesidades de información de los agricultores y los campesinos.

\section{Obras CONSUltadas}

Ashill, Nicholas J., "Defining the information needs of senior marketing executives: an exploratory study”, pp. 52-61, en Qualitative Market Research: An International Journal, Vol. 4, núm. 1 (2001). 
Baja California: la frontera Mexicana para negocios de éxito, http:// www.investinbaja.gob.mx/industrias/alimentos.htm 27-sep-2008.

Baja California: la frontera mexicana para negocios de éxito : Ruta del vino, http://www.investinbaja.gob.mx/estilo/recreacion.htm\#06 27-sep-2008.

Calva González, Juan José, "Las necesidades de información de los usuarios en la planeación bibliotecaria”, en Biblioteca Universitaria: Boletín Informativo de la Dirección General de Bibliotecas, Vol. 6, Núm. 1, 1991.

Las necesidades de información: su naturaleza, su manifestación y detección, México, el autor, 1988, 247 p. (Tesis de Maestría), UNAM, Facultad de Filosofía y Letras.

Caminata mensual a San Antonio de las Minas en Ensenada, http://bajacalifornia.travel/caminata-mensual-a-san-antonio-de-las-minasen- ensenada/ 1-sep-2008.

Castillo Sánchez, Octavio, "Técnicas de recolección de información más utilizadas en los estudios de usuarios”, en Boletín del Departamento de Bibliotecología, Núm. 16 (1987).

Devadason, J. W., "A methodology for identification of information needs of users”, en IFLA Journal, Vol. 23, Núm. 1, 1997.

Enciclopedia Hispánica, Barcelona, 2a. Ed., Editorial Barsa-Planeta, 2003, T. 14.

Enciclopedia universal ilustrada: europea americana, Madrid, EspasaCalpe, 1996.

Guzmán Peredo, Miguel, El vino en México, http://www.mexicodesconocido.com.mx/notas/744-El-vino-en-México, 2-feb-2009.

Harris, Colin, "Necesidades de los usuarios y mercado de la información: su importancia en el curriculo de las escuelas de bibliotecología”, en Revista interamericana de Bibliotecología, Vol. 9, Núm. 1, 1986, p. 15.

Kuruppu, Pali U., "Understanding the information needs of academic scholars in agricultural and biological sciences", en The Journal of Academic Librarianship, Vol. 32, Núm. 6, 2006, p. 609.

Melgar Gil, Luis Tomás, La enciclopedia del vino, Madrid, Ramón Llaca, 2008.

Monte Xanic, http://www.montexanic.com.mx/inicio.html 2-ago-2008.

Nason, Lisa, "The farmers in the library: information needs of farmers and how the rural public library can fulfill them", 19-45 pp., en Rural Library, Vol. 27, Núm. 2, 2007.

Profesionales del vino mexicano y de las vinícolas en México, http:// vinosdemexico.homestead.com/vinicolas.html 1-jun-2008.

Restaurantes de Mexico.com.mx La vendimia: una tradición de la industria vinícola, http://www.restaurantesdemexico.com.mx/379/ Vinos_y_Bebidas_La_Vendimia__Una_Tradicion_En_La_Industria_Vinicola.html\# 6-ago-2008.

Sanz Casado, Elías, Manual de estudios de usuarios, Madrid, Fundación Germán Sánchez Ruipérez, 1994. 
Santos Rosas, Antonia y Juan José Calva González, "Identificación de las Necesidades de información de los usuarios: un estudio", en Revista: Documentación de Ciencias de la Información, Núm. 20, 1997, pp. 207-224.

Sistemas Producto: Perspectivas del mercado de la uva, http://www. campomexicano.gob.mx/portal_sispro/index.php?portal=uva, 26-sep-2008.

“Terminología relacionada con los estudios de usuarios", en Seminario Latinoamericano sobre Formación de Usuarios de la Información y Estudios de Usuarios, México, CUIB, 1997.

Valle de Guadalupe en Wikipedia, http://es.wikipedia.org/wiki/Valle_de_Guadalupe_(Baja_California) 30-junio-2008.

Vinos L. A, Cetto: vinos con nombre y apellido, http://www.lacetto. com/28-ago-2008. 
